\title{
Nivel de conocimiento de las madres sobre contaminación de plomo en niños menores de 5 años
}

\author{
Castro Solórzano Fiorella ${ }^{1 \mathrm{a}}$, Poma Arroyo Rosario ${ }^{1 \mathrm{~b}}$, Salcedo Santos Christy ${ }^{1 \mathrm{c}}$, Fernández María Ángela ${ }^{2 \mathrm{~d}}$
}

\section{RESUMEN}

Objetivo: determinar el nivel de conocimiento de las madres sobre la contaminación de plomo en niños menores de 5 años, 2014. Material y Métodos: estudio cuantitativo, descriptivo y de corte transversal. La población de estudio estuvo conformada por 80 madres de niños menores de 5 años de la localidad de Paragsha - Cerro de Pasco-Perú quienes cumplieron con los criterios de inclusión y exclusión. La recolección de datos se realizó a través de un cuestionario de 25 preguntas que fue elaborado por las investigadoras y validado a través de un juicio de expertos. Para determinar la confiabilidad se realizó una prueba piloto en 15 madres de niños menores de 5 años, los datos obtenidos fueron codificados, ingresados, analizados porcentualmente en el programa de Excel y presentados en tablas de doble entrada. Resultados: los conocimientos de las madres sobre contaminación por plomo se encuentra en categoría regular en un 56.25\%. Conclusiones: En su mayoría las madres tienen conocimiento regular sobre contaminación por plomo en niños menores de 5 años.

PALABRAS CLAVES: conocimientos, contaminación, plomo.

\section{Level of knowledge of mothers on lead contamination in children under 5 years}

\begin{abstract}
Objective: determine the level of knowledge of mothers about lead contamination in children under 5 years, 2014 . Material and Methods: quantitative Study, descriptive and cross-sectional. The study population consisted of 80 mothers of children under five years of the town of Paragsha - Cerro de Pasco who met the criteria for inclusion and exclusion. Data collection was conducted through a questionnaire of 25 questions which was developed by the researchers and validated by an expert opinion. To determine the reliability a pilot test was conducted in 15 mothers of children under five years, the data were coded, entered, analyzed percentage in the Excel program and presented in two-way tables. Results: the knowledge of mothers about lead contamination is in regular category in $56.25 \%$. Conclusions: mostly mothers have regular knowledge about lead contamination in children under 5 years.
\end{abstract}

KEY WORDS: knowledge, pollution, lead.

\footnotetext{
Licenciada en enfermería

Maestra en Gestión de los Servicios de Salud.

Clínica del Trabajador Lima - Perú, ${ }^{\mathrm{b}}$ Medical Asistant Lima - Perú, ${ }^{\mathrm{c}}$ Integra Medica Lima - Perú, ${ }^{\mathrm{d}}$ Hospital Nacional Arzobispo Loayza
} 


\section{INTRODUCION}

El plomo es un elemento muy tóxico, sus partículas pueden ser re-suspendidas por el viento hacia el suelo y de este modo el elemento puede ingresar al organismo humano por vía respiratoria, vía digestiva y vía cutánea (1).

La contaminación por plomo es uno de los problemas ambientales más importantes que afectan al mundo (2). Y surge cuando se produce un desequilibrio, como resultado de la adición de este metal al medio ambiente, debido a los diferentes procesos productivos del hombre, causando efectos adversos en la población.

A nivel mundial el Perú es el cuarto productor de plomo y el primero en América Latina; Siendo Pasco el primer productor a nivel nacional, en mayo del 2012 el Ministerio del ambiente declara en emergencia ambiental a 6 localidades del distrito de Simón Bolívar, provincia y departamento de Pasco, entre ellos Paragsha (3).

Paragsha se encuentra adyacente a la planta concentradora de metales y tajo abierto, las viviendas colindantes al centro de salud son antiguos campamentos mineros, donde también existen invasiones hacia ambos lados del cerro. Los cuales se encuentran a lo largo de una vía de transporte de carga de minerales.

Según un informe de la DIRESA Pasco - Sistema de vigilancia epidemiológica; el $80 \%$ de la población evaluada reside igual o mayor a 6 años en la localidad de Paragsha, mientras que el $10 \%$ reside con un tiempo no menor a 6 meses y el resto es migrante (4).

Asimismo en relación al tiempo de exposición a la contaminación por plomo de la localidad oscila entre 5 y 8 años. Siendo el suelo el principal factor de riesgo ya que la concentración del plomo es 3.9 veces mayor a lo permitido (5). Por otro lado según el informe realizado por el Centro Nacional de Salud Ocupacional y Protección para el Ambiente para la Salud (CENSOPAS) del Instituto Nacional de Salud en coordinación con la DIRESA Pasco, reportan que los niños menores de 5 años de la comunidad antes mencionada, tienen plomo en sangre entre los niveles 10 a mayor a $30 \mathrm{mg} / \mathrm{dl}$, de acuerdo a la clasificación y recomendación de la Guía Práctica clínica para el manejo de pacientes con intoxicación por plomo (6).

Siendo los niños más vulnerables que los adultos a presentar efectos en la salud por exposición a este metal a bajas concentraciones. Ya que ellos están más cerca del suelo e ingieren partículas del mismo, así como polvo doméstico contaminado, en mayores cantidades que los adultos, en especial los niños con hábito de pica (comer tierra, morder lápices y juguetes) (7) y a la vez su cerebro, sistema nervioso central y capacidades intelectuales están en una etapa muy importante de su desarrollo lo que conlleva a ingerir y absorber el plomo con más facilidad. Asimismo la absorción de plomo aumenta cuando el aporte de otros minerales y proteínas en la dieta es inadecuado. Así aquellos con deficiencia de hierro, calcio y zinc están en mayor riesgo de intoxicación (8).

Para poder identificar los factores de riesgo y prevenir complicaciones a través de la educación sanitaria y comunicación de riesgos, es importante fomentar la responsabilidad de la familia y las autoridades involucradas en este tema, así se facilitará la promoción, difusión de la información y orientación en las instituciones y organizaciones. Todo ello para dar a conocer y/o fortalecer conocimientos con el fin de evitar complicaciones futuras en los niños, que se encuentran en pleno desarrollo de sus capacidades físicas, cognitivas e intelectuales.

Por lo que enfermería tiene como rol primordial el seguimiento o control de la salud del usuario, identificando los factores de riesgo que están presentes en su entorno, a fin de realizar acciones de promoción de hábitos de vida saludable y prevención de complicación, tanto en el hogar como en la comunidad (9).

\section{MATERIAL Y METODOS}

Enfoque cuantitativo de tipo descriptivo de corte transversal. Se realizó en 6 clubes de madres del vaso de leche los cuales se encuentran ubicados en el Centro poblado menor Paragsha, Cerro de Pasco - Perú. Dicha localidad se encuentra adyacente a la planta concentradora de metales, en su interior y en los alrededores de dicha empresa transitan volquetes, trenes y camiones pesados que transportan el material procedente de la mina subterránea y del tajo abierto siguiendo su recorrido por las principales avenidas de la localidad, hacia Lima u otras provincias.

La población estuvo constituida por 80 madres de niños menores de 5 años, que cumplieron con siguientes criterios de selección.

\section{Criterio de inclusión:}

- Madres que voluntariamente aceptaron participar en el estudio y firmaron el consentimiento informado. 


\section{Criterio de exclusión:}

- Madres de niños que tenían alguna enfermedad previamente diagnosticada.

- Madres que tenían dificultades para responder el instrumento de estudio.

La recolección de datos se realizó a través de una encuesta y se aplicó un cuestionario a las madres de forma anónima, estructurado en 2 partes.

I. Datos sociodemográficos: grado de instrucción, edad de la madre, número de hijos, tiempo de residencia en la localidad.

II. Conocimientos sobre contaminación, factores de riesgo y prevención, con un total de 25 preguntas, relacionadas a las dimensiones: Factores de riesgo de la contaminación, prevención de la contaminación, contaminación por plomo.

Cada una de las preguntas con cinco alternativas de respuesta y una sola respuesta correcta, cada respuesta tuvo un valor de 1 punto, haciendo un total de 25 puntos que se clasificaron usando la escala de Estaninos en nivel de conocimiento sobre contaminación por plomo:

$$
\begin{gathered}
\text { Bueno }=19-25 ; \text { Regular }=14-18 ; \\
\text { Deficiente }=0-13 \text { puntos }
\end{gathered}
$$

El instrumento se sometió a juicio de expertos, en el que participaron 8 profesionales: 5 enfermeras, 2 médicos y 1 psicólogo, lo que permitió realizar ajustes en la redacción de algunas preguntas.

Asimismo se aplicó una prueba piloto a 15 madres con criterios de selección similares a la del estudio que no formaron parte de la muestra y que residían en la localidad de San Juan, aledaño a la localidad de Paragsha.

Después de obtener la autorización de la DUITC, la Facultad de Enfermería y del Comité de ética de la Universidad Peruana Cayetano Heredia.

- En la primera sesión se realizó la coordinación con la presidenta del comité central del vaso de leche de la localidad de Paragsha para solicitar el permiso correspondiente para dar inicio a la ejecución del proyecto de investigación, asimismo, se solicitó una reunión con las socias de los clubes de madres del vaso de leche.

- En la segunda sesión se llevó a cabo la reunión con las socias de los comités del vaso de leche en la cual se les explicó los objetivos del proyecto, se les solicitó su participación voluntaria en el estudio de investigación a través del consentimiento informado, este fue un proceso anónimo sin perjuicio alguno para los entrevistados.

- Aceptada la participación se realizó la aplicación del instrumento con una duración aproximada de $30 \mathrm{mi}-$ nutos.

Una vez recolectado los datos se codificaron y procesaron en una base de datos en Microsoft Excel 2013. Los resultados obtenidos fueron analizados porcentualmente en el programa de Excel y presentados en tablas estadísticas de doble entrada.

\section{RESULTADOS}

Se pudo observar que del total de madres encuestadas, el 56,25\% tienen nivel de conocimiento regular sobre la contaminación por plomo en niños menores a 5 años y el menor porcentaje $17,50 \%$ nivel bueno (Tabla 1 ).

Por otro lado, se obtuvo que del total de madres encuestadas, el 52,50\% tienen nivel de conocimiento regular sobre los factores de riesgo, y un 7,50 \% tiene nivel bueno (Tabla 2).

Del total de madres encuestadas, un 43,75\% tienen nivel de conocimiento regular sobre prevención, y un 26,25\% tiene nivel bueno (tabla 3 ). Por otro lado, presentan grado de instrucción superior el 70,8\% tienen conocimiento regular sobre contaminación por plomo, asimismo del total

Tabla 1. Nivel de conocimiento de las madres sobre contaminación por plomo, localidad de Paragsha - Cerro de Pasco, 2014.

\begin{tabular}{lcr}
\hline $\begin{array}{l}\text { Nivel de conocimiento de las madres } \\
\text { sobre contaminación por plomo }\end{array}$ & $\mathrm{N}^{\circ}$ & $\%$ \\
\hline Bueno & 14 & 17,50 \\
Regular & 45 & 56,25 \\
Deficiente & 21 & 26,25 \\
Total & 80 & 100,00 \\
\hline
\end{tabular}

Tabla 2. Nivel de conocimiento de las madres sobre los factores de riesgo de contaminacion por plomo, Paragsha - Cerro de Pasco, 2014.

\begin{tabular}{lcr}
\hline $\begin{array}{l}\text { Nivel de conocimiento de las madres } \\
\text { sobre factores de riesgo }\end{array}$ & $\mathrm{N}^{\circ}$ & \multicolumn{1}{c}{$\%$} \\
\hline Bueno & 6 & 7,50 \\
Regular & 42 & 52,50 \\
Deficiente & 32 & 40,00 \\
Total & 80 & 100,00 \\
\hline
\end{tabular}


de madres que tiene grado de instrucción secundaria el $52,0 \%$ tiene nivel de conocimiento regular (tabla 4 ), y del total de madres entre las edades de 31 a 40 años el $66,6 \%$ tiene nivel de conocimiento regular sobre contaminación por plomo (tabla 5).

\section{DISCUSIÓN}

Con el objetivo de determinar el nivel de conocimiento de las madres sobre contaminación de plomo en niños menores de 5 años, de la localidad de Paragsha-Cerro de

Tabla 3. Nivel de conocimiento de las madres sobre la prevención de contaminacion por plomo, Paragsha - Cerro de

\begin{tabular}{lcc}
\multicolumn{3}{c}{ Pasco, 2014. } \\
$\begin{array}{llc}\text { Nivel de conocimiento de las } \\
\text { madres sobre prevención }\end{array}$ & $\mathrm{N}^{\circ}$ & \\
\hline Bueno & 21 & 26.25 \\
Regular & 35 & 43.75 \\
Deficiente & 24 & 30.00 \\
Total & 80 & 100.00 \\
\hline
\end{tabular}

Pasco, se desarrolló una investigación en una población muestral de 80 madres de familia que tenían entre 20 a 50 años de edad, que la mayoría contaba con educación secundaria siendo el $62,50 \%$, y tenían entre 1 - 2 hijos siendo el 51,25\% (anexo 5); encontrando similitud con Benavente Lipa L. quién en su investigación sobre conocimientos de madres en el estado de salud del niño encontró que las madres tienen grado de instrucción secundaria 60 $\%$ (10). Por otro lado encontrándose que en relación a los conocimientos de las madres sobre contaminación de plomo, la mayoría de las madres tenían nivel de conocimiento regular sobre la contaminación de plomo en niños menores a 5 años y una menor parte conocimiento bueno (Tabla 1). Teniendo en cuenta que más de la mitad de las madres encuestadas cuenta con educación secundaria completa eso nos permite saber que cuentan con una herramienta importante que es la educación y que esta las ayuda para poder recibir información sobre el tema. Por el contrario si a pesar de ello no ponen en práctica la información y cuidados que deben tener hacia sus hijos se basa más en un tema actitudinal más que de nivel de conocimiento.

TABLA 4. Nivel de conocimiento de las madres sobre contaminación por plomo según grado de instrucción, Paragsha - Cerro de Pasco, 2014.

\begin{tabular}{ccccccccc}
\hline \multirow{2}{*}{$\begin{array}{c}\text { Grado de } \\
\text { Instrucción }\end{array}$} & \multicolumn{3}{c}{ Nivel de conocimiento de las madres sobre contaminación por plomo } & \multirow{2}{*}{ Total } \\
\cline { 2 - 6 } & \multicolumn{2}{c}{ Bueno } & \multicolumn{2}{c}{ Regular } & \multicolumn{2}{c}{ Deficiente } & & \\
\hline & $\mathrm{N}^{\circ}$ & $\%$ & $\mathrm{~N}^{\circ}$ & $\%$ & $\mathrm{~N}^{\circ}$ & $\%$ & $\mathrm{~N}^{\circ}$ & $\%$ \\
Primaria & 0 & 0 & 2 & 33,3 & 4 & 66,7 & 6 & 100 \\
Secundaria & 10 & 20,0 & 26 & 52,0 & 14 & 28,0 & 50 & 100 \\
Superior & 4 & 16,7 & 17 & 70,8 & 3 & 12,5 & 24 & 100 \\
& 14 & 17,5 & 45 & 56,3 & 21 & 26,3 & 80 & 100 \\
\hline
\end{tabular}

TABLA 5. Nivel de conocimiento de las madres sobre contaminación por plomo según edad, Paragsha - Cerro de Pasco, 2014.

\begin{tabular}{|c|c|c|c|c|c|c|c|c|}
\hline \multirow[t]{3}{*}{ Edad } & \multicolumn{6}{|c|}{$\begin{array}{l}\text { Nivel de conocimiento de las madres sobre contaminación por } \\
\text { plomo }\end{array}$} & \multirow{2}{*}{\multicolumn{2}{|c|}{ Total }} \\
\hline & \multicolumn{2}{|c|}{ Bueno } & \multicolumn{2}{|c|}{ Regular } & \multicolumn{2}{|c|}{ Deficiente } & & \\
\hline & $\mathrm{N}^{\circ}$ & $\%$ & $\mathrm{~N}^{\circ}$ & $\%$ & $\mathrm{~N}^{\circ}$ & $\%$ & $\mathrm{~N}^{\circ}$ & $\%$ \\
\hline 20 a 30 años & 3 & 12,5 & 14 & 58,3 & 7 & 29,1 & 24 & 100 \\
\hline 31 a 40 años & 7 & 25,9 & 18 & 66,6 & 2 & 7,4 & 27 & 100 \\
\hline \multirow[t]{2}{*}{41 a 50 años } & 4 & 13,7 & 13 & 44,8 & 12 & 41,3 & 29 & 100 \\
\hline & 14 & 17,5 & 45 & 56,3 & 21 & 26,3 & 80 & 100 \\
\hline
\end{tabular}


Según la guía de prevención, diagnóstico, tratamiento y vigilancia epidemiológica de las intoxicaciones infantiles por plomo afirma que la absorción de plomo aumenta cuando el aporte de otros minerales y proteínas en la dieta es inadecuado. Así aquellos con deficiencia de hierro, calcio y zinc están en mayor riesgo de intoxicación. Siendo los niños más vulnerables que los adultos a presentar efectos en la salud por exposición a este metal a bajas concentraciones. Esto se deberá en parte a que los niños están más cerca del suelo, pasan más tiempo en él, e ingieren partículas del mismo, así como polvo doméstico contaminado, en mayores cantidades que los adultos, en especial los niños con hábito de pica (comer tierra, morder lápices y juguetes) (7) y a la vez su cerebro, sistema nervioso central y capacidades intelectuales están en una etapa muy importante de su desarrollo lo que conlleva a ingerir y absorber el plomo con más facilidad (8).

Considerando entonces que el comportamiento esta guiado en parte por el conocimiento que las personas tienen sobre situaciones específicas es relevante citar el último informe realizado por el Ministerio de Salud, donde se muestra que una de los principales factores de riesgo es la falta de conocimiento de las madres e información limitada sobre los riesgos puede conllevar el habitar en un entorno contaminado (4), tal como se observa en las madres encuestadas que en su mayoría muestran conocimiento regular y una mínima parte conocimiento bueno (Tabla 2).

Los factores de riesgos están relacionadas con las fuentes de contaminación; trabajar y/o habitar en zonas de exposición, desnutrición, edad menor a 6 años, deficiente condición sanitaria de la vivienda, malos hábitos higiénicos y malas condiciones e higiene y seguridad ocupacional; por lo que la exposición intensa a altas concentraciones durante un tiempo, produce una intoxicación aguda con compromiso del sistema nervioso central, sistema nervioso periférico, hematopoyético, renal, metabólico entre otros; especialmente en los niños menores a 5 años quienes son los más sensibles ya que suelen ingerir y absorber el plomo con más facilidad (11).

En relación al nivel de conocimiento de las madres sobre la prevención en la localidad de Paragsha, se observa que un gran porcentaje tiene conocimiento regular (Tabla 3), ya que la prevención por plomo se puede hacer a través de una serie de estrategias como la eliminación y/o control de la exposición ambiental, educación sanitaria y comunicación de riesgos, que busca la modificación de los hábitos que conllevan riesgos de contaminación en niños, siendo los más importantes para el bienestar y la mejora de los estilos de vida, higiene personal, lavado de manos, chupones y juguetes para reducir asi la exposición e intoxicación por plomo. Cambiar de ropa después de llegar del trabajo, esto seguidamente de la higiene de la vivienda, los pisos y las superficies, mantener siempre la casa libre de polvo, cubrir alimentos y depósitos de agua, por último mejorar el estado nutricional y consumir el agua hervida (7).

En relación al nivel de conocimiento de las madres sobre contaminación de plomo según grado de instrucción, se encontró que la mayoría de las madres encuestadas tienen grado de instrucción secundaria; asimismo la mayor parte de ellas tienen un nivel de conocimiento regular (Tabla 4), encontrando similitud con Benavente L (2012). quién encontró que las madres tienen grado de instrucción secundaria $60 \%$ y que existe asociación significativa entre el grado de instrucción y el nivel de conocimiento de las madres de familia (10).

Según el estudio de Correa E. y Guerra S (2011). donde evidencian que un $52 \%$ de las madres tienen instrucción secundaria (completa e incompleta), el 38\% tienen instrucción primaria (completa e incompleta), los datos expresan el predominio del grado de instrucción de primaria y secundaria, lo que significa que las madres podrían tener dificultades para adquirir los conocimientos científicos básicos de salud interfiriendo así en la prevención y tratamiento de las enfermedades y en general en el cuidado de la salud del niño (12), teniendo en cuenta el último censo realizado por el INEI, el $41 \%$ de la población tiene secundaria completa y resaltando que el conocimiento no es innato, sino es la suma de hechos y principios que se adquieren a lo largo de la vida como resultado de la experiencia y el aprendizaje del sujeto. Por ende la carencia de los conocimientos generará comportamientos errados y más aun sabiendo que la población se encuentra expuesta a la contaminación ambiental debido a la explotación minera.

En relación a nivel de conocimiento de las madres sobre contaminación de plomo según edad; dentro de éste marco se encontró que del total de madres; la mayor parte de las encuestadas abarcan entre edades de 31 a 40 y presentaron un nivel de conocimiento regular (Tabla 5), ellas son económicamente activas de mayor potencial de desarrollo familiar, esto se debe a la falta de promoción por parte del sector salud ya que actualmente es un problema de Salud Pública.

Además; existen estrategias, programas y guías sobre intoxicación plomo, donde una de sus recomendaciones 
como medida preventiva se encuentra la educación sanitaria y comunicación de riesgos: modificación de los hábitos, higiene personal y de vivienda (1), sin embargo se encontró en los tres grupos de madres según edad en su mayoría no se encuentran informadas, puesto que presentan conocimientos de forma regular, y si lo están pues no los aplican lo cual quiere decir que no existe una buena sensibilización de parte del sector correspondiente.

Teniendo en cuenta que esta población está dedicada a la minería, siendo para los hombres la principal actividad económica y por lo que son las madres quienes asumen toda la responsabilidad del cuidado de sus hijos, sin embargo las organizaciones no muestran interés de trabajar en ellas y/o brindarles información sobre el problema, tan solo se centran en la sintomatología y tratamiento de aquellos niños intoxicados con plomo más no en controlar los factores de riesgo asociados exponiéndolos a una mayor contaminación.

\section{REFERENCIAS BIBLIOGRAFÍCAS}

1. Resolución Ministerial 15 de junio 2007 del Ministerio de Salud. Guía de Práctica clínica para el manejo de pacientes con intoxicación por plomo, Lima; 2007. Disponible en: Www.sis.gob.pe/ipresspublicas/ normas/pdf/minsa/GUIASPRACTICAS/2007/ RM511_2007.pdf (acceso 16 de septiembre 2014).

2. Franco D. Contaminación por Plomo. Informe elaborado por la Comisión de Salud Ocupacional del Sindicato Médico del Uruguay. 2010. Disponible en : www.smu.org.uy/ sindicales/resoluciones/informes/plomo.pdf (acceso $13 \mathrm{de}$ septiembre 2014)

3. Ministerio de Energía y Minas. Producción minera. Julio 2011. Disponible en: http://es.scribd.com/doc/59689103/ Produccion-Minera (acceso 22 de noviembre del 2013).

4. Ministerio del Ambiente. Nota informativa de la Emergencia Ambiental de seis localidades de la Provincia de Pasco. Noviembre 2012, Lima- Perú: Ministerio de salud.

5. Ministerio de salud DIGESA. Intoxicación por plomo ambiental. 2008. Disponible en:

www.minsa.gob.pe/portada/est_san/cmposq.htm\# (acceso 11 de Octubre del 2013).

6. Centro Nacional de Salud Ocupacional y protección para el ambiente para la salud (CENSOPAS). Niveles de Exposición al Plomo y Cadmio en Niños menores de 12 años y Gestantes de 6 comunidades del Pasco. Informe del Instituto Nacional de Salud- Ministerio de salud, Mayo - Junio 2012.

7. Ministerio de salud Presidencia de la Nación. Guía de prevención, diagnóstico, tratamiento y vigilancia epidemiológica de las intoxicaciones infantiles con plomo. Programa nacional de prevención y control de las intoxicaciones. Argentina; 2013. Disponible en:

w w w. m s a l.gov. ar/i mages/stories/bes/ graficos/0000000293cnt-guia_intoxicaciones_con_ plomo_2013.pdf (acceso 05 de julio 2014).

8. Sanín L, González-Cossío T, Romieu I. Acumulación de plomo en hueso y sus efectos en la salud. Salud pública de México. México. 40 (4). Disponible: www.bvsde.paho.org/ bvstox/e/fulltext/acumul/acumul.pdf (acceso 5 de julio del 2014).

9. Guerrero M. Impacto de la Exposición a Plomo en los Niños del Callao - Perú. Diagnostico e Intervención Psicoeducativa. Lima - Perú; 2009. Primera edición Fundación Cayetano Heredia.

10. Benavente L. Nivel de Conocimientos de las Madres y su relación con el estado de salud bucal del niño menor de cinco años de edad. Revista Odontología Sanmarquina; 2012. 15(1). Lima - Perú. Disponible en: http://sisbib. unmsm.edu.pe/bvrevistas/odontologia/2012_n1/pdf/ a05v15n1.pdf (acceso 09 de Julio 2015)

11. Moreyra NG; Prado R. Relación entre la contaminación ambiental por plomo y el crecimiento y desarrollo de niños de 4-5 años en dos instituciones educativas iníciales en la Región Callao Lima. Tesis de licenciatura. Facultad de enfermería de la escuela de sanidad naval Universidad peruana Cayetano Heredia. 2010.

12. Correa E. Guerra S. Nivel de Conocimientos Relacionado con Prácticas sobre Infecciones Respiratorias Agudas en Madres de Niños Menores de 5 años. Centro de Salud Morales. Octubre -Diciembre 2011. Tesis de licenciatura. Universidad Nacional de San Martin Facultad Ciencias de la Salud Escuela Profesional de Enfermería. 2012. Disponible en: http://www.unsm.edu.pe/spunsm/ archivos_proyectox/archivo_103_Binder1.pdf (acceso 09 de Julio 2015).

\section{Correspondencia:}

Salcedo Santos Christy

Correo electrónico: 1i_kity23@hotmail.com

Fecha de Recepción: 20 de Octubre del 2015.

Fecha de aceptación: 11 de Noviembre del 2015. 